\title{
A visits programme for forensic senior registrars
}

\author{
Akintunde Akinkunmi
}

\begin{abstract}
As part of the academic programme for senior registrars in forensic psychiatry working in the North Thames Region, a series of visits to outside institutions of special relevance was organised. These visits form part of a larger academic programme which includes consultant tutorials, journal clubs and case conferences. Between May 1995 and October 1996 the author was the senior registrar in charge of organising the programme and this report is intended to share the experiences gained in visiting these other institutions.

The outside institutions listed below were visited by the group and a brief description of the visit follows each institution. (For readers considering a visits programme, a list of addresses and contact persons for the institutions listed below is available from the author on request.)
\end{abstract}

\section{Mental Health Unit (formerly C3 Division) of the Home Office \\ This provided a useful insight into the workings of this Unit, which has direct relevance to every forensic psychiatrist practising in England and Wales. It was helpful in understanding the procedures followed in decision-making and also provided for the making of valuable contacts which have made subsequent dealings with that division smoother and quicker. The officers working in this division were all very helpful, easy to approach and talk to and their hospitality was excellent.}

\section{HMP Grendon Underwood}

This, as most readers will be aware, is the only prison in England and Wales where treatment of people with mental disorders, principally personality disorders, is as much an objective as is their detention. We were shown around by the Senior Medical Officer and were given the opportunity to sit in on one of the group sessions. It was particularly interesting to watch the dynamics of the group, particularly the ease with which they discussed their offending behaviour, the reasons for it, as well as the way in which such revelations were received and responded to by other members of the group.

Directorate of Healthcare, Headquarters of HM Prison Service

Given the close relationship between practising forensic psychiatrists and the Healthcare service of the prison service, this visit was particularly appropriate. It provided a useful insight into some of the difficulties under which the providers of healthcare within the prison service have to work, particularly in the prevailing culture that treatment comes a poor second to security. The group came away with an increased sympathy for any subsequently experienced shortcomings in the delivery of psychiatric care within the prison healthcare system.

\section{Carstairs Hospital, Scotland}

This was the first visit undertaken to an institution outside the jurisdiction of the Mental Health Act 1983 in operation in England and Wales. It was a useful introduction to understanding the provision of forensic psychiatric care under differing legislative arrangements.

\section{Van der Hoeven Kliniek, Utrecht,}

The Netherlands

The contrast in mental health legislation, which was first apparent at Carstairs, was highlighted even more starkly during the visit to the Van der Hoeven Kliniek. The staff were extremely receptive and took a lot of time and trouble to explain how the unit functions within Dutch legislation. Of particular interest was the TBS system which bears a remarkable similarity to the hybrid order for psychopathic disorders currently under discussion in England (the Van der Hoeven Kliniek only treats patients with a diagnosis of psychopathic disorder). It is an extremely well staffed and resourced unit with industrial therapy facilities of a commerical standard-indeed, patients at the unit have secured commercial contracts for the goods they produce. The discussions about the TBS system were full and frank, and had the effect among the group of 
increasing either support for, or opposition to, the hybrid order in the UK.

\section{The Medical Protection Society}

This was a visit much closer to home but no less interesting and informative. Again, the group was very well received and were able to discuss at length some of the concerns held by members of the group in relation to forensic psychiatric practice. The group had the opportunity of discussing fully and frankly with the staff at the MPS ways in which forensic psychiatrists might fall foul of the laws concerning negligence.

\section{The Central Mental Hospital, Dundrum, Dublin, Ireland}

This perhaps was one of the most challenging of the visits undertaken by the group. By the admission of the staff working there, the absence of updated mental health legislation can cause great difficulty in the provision of forensic psychiatric care to a standard which they would like to provide, and the relative lack of resourcing only makes this picture worse. This is, however, a prevailing mood of change and it is hoped that in the not too distant future the situation will more closely match the aspirations of the staff who work within forensic psychiatry in Dublin.

\section{The Black Museum, Scotland Yard}

This is definietly not for the faint-hearted as it is difficult to imagine a greater collection of the macabre, the exotic, and the downright terrifying in one place. It does, however, expose one to the huge repertoire of black humour stories which policemen in general, and the genial curator of this museum in particular, see as an essential way of coping with what can be a very grim and stressful job. Unfortunately access to the museum is restricted and the group is indebted to its curator for permitting us to see it and showing us around so enthusiastically.

\section{The Kirby Forensic Psychiatric Center, New York}

This was by far the most ambitious visit attempted, but it was well worth the trip. The Kirby center is one of three maximum security hospitals in the state of New York and is located on an island off Manhattan. It has 165 patients in seven wards with a maximum of 25 patients on each ward. It is run by ward-based clinical teams, each consisting of a full-time psychiatrist, a full-time psychologist, social worker and one trained ward nurse. There are also four Secure Hospital Treatment Aides (SHTAs). These latter are not trained nurses. It covers a catchment area of the five boroughs of the city of New York and $80 \%$ of its patients are referrals from the court system, split between those who are considered unfit to plead and those who have been found not guilty by reason of insanity (NGRIs). One-fifth of its referrals are transfers from general psychiatric hospitals for patients whose behaviour is so di iturbed that i hey canr ot be coped with in a non-secure settinf. There are no medium secure units, as is the practice in the UK.

The hospital also has a proper court room, used for hearings into renewal of detention/ discharge of detained patients in the categories described above, as well as for hearings into the need to medicate even detained patients against their will. A judge attends all these heaings and there is legal representation of the patient, the District Attorney's office (the local equivalent of the Crown Prosecution Service) and, unusually, the hospital itself. There is no provision for escorted or unescorted community parole, and patients who have been admitted as unfit to plead, and who are consequently deemed to have become fit to plead, may be returned to prison custody by the simple expedient of placing a telephone call to the prison who come and collect the patient.

The staff at the Kirby Center are always keen to receive visitors from overseas and to share experiences of forensic psychiatric practice under different legislative settings to the mutual benefit of all concerned.

\section{Useful tips}

Draw up a timetable of the available dates and consult widely with colleagues about places they would like to visit. These should then be allocated to a specific date.

Give as much notice as possible to the receiving institution, especially those that are either some distance away or provide a greater degree of security than medium security. These institutions usually require a list of names of those who will be attending and taking along some proof of identity with a photograph is essential.

For those institutions overseas it is essential to keep costs down. Negotiate with your study leave budget holder about what would be an appropriate level of expenses and if, for instance. planning to visit New York, it may be helpful to agree to have only transport costs rather than both transport and subsistence costs reimbursed (a return plane fare to New York costs no more than what one would reasonably expect to pay for a good management course). If a large party is travelling it may be helpful to bulk-book tickets as you may be able to negotiate a better discount if there are more people travelling. 
Constant liaison with colleagues is essential, especially to ensure that the available dates are kept free and to remind people of dates set for visits to particular institutions. Interest in visiting will be increased if a programme for the visit is attached to all correspondence.

Closely involve the scheme organiser in the planning of all visits. Their assistance in getting costs reimbursed is invaluable.

Where possible, try to ensure common travelling and accommodation arrangements as this makes it easier to coordinate the visit and ensures that everybody arrives in the right place at the right time.

Organising these visits has been a rewarding experience, although it can involve hard work especially in trying to coordinate a group of senior registrars working in different institutions.

Akintunde Akinkunmi, Senior Registrar in Forensic Psychiatry, Camlet Lodge Regional Secure Unit, Chase Farm Hospital, Enfield, Middlesex EN2 8JL

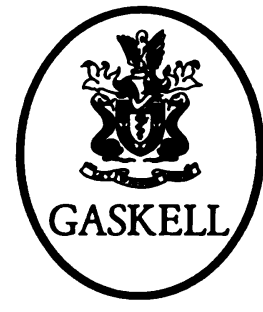

\section{Seminars in Practical Forensic Psychiatry}

\section{Edited by Derek Chiswick \& Rosemary Cope}

Seminars in Practical Forensic Psychiatry is a concise account of the specialty from a strongly practical perspective. It systematically describe the relationship between psychiatric disorders and offending, with detailed discussion of the criminal justice system, court proceedings, mental health legislation, dangerousness, prison psychiatry, and civil issues. There are boxes summarising key points, illustrative case examples, and sample court reports. It is up-to-date, with references to the Reed Report, the Clunis Inquiry, supervision registers and recent legislation. Career guidance and a chapter on ethical issues are included. This book will be invaluable for general psychiatrists and for trainees in forensic psychiatry, as well as those other health and social work professionals having contact with mentally disordered offenders, and those who are part of the criminal justice system. Price EI 7.50, 359pp., 1995, ISBN 0902241788

Available from good bookshops and from the Publications Department, Royal College of Psychiatrists, 17 Belgrave Square, London SWIX 8PG (Tel. O17I-235 235I, extension 146) 